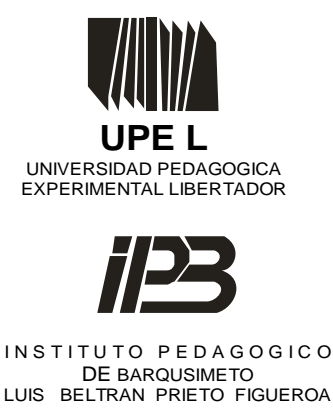

DE BARQUSIMETO
LUIS BELTRAN PRIETO FIGUEROA

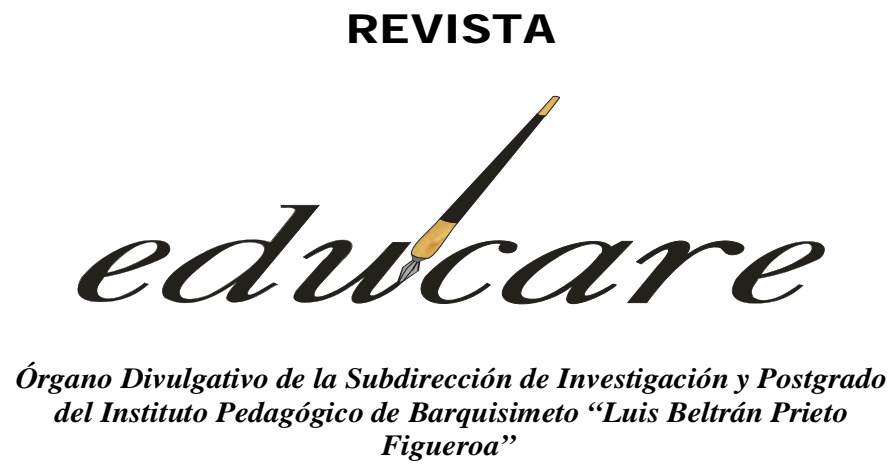

Figueroa"

BARQUISIMETO - EDO. LARA - VENEZUELA

NUEVA ETAPA

FORMATO ELECTRÓNICO

DEPOSITO LEGAL: ppi201002LA3674

Volumen $18 \mathrm{~N}^{\circ} 1$

ISSN: $2244-7296$

Enero - Abril 2014

\title{
LAS GEMAS DE LA ORGANIZACIÓN INNOVADORA: CÓMO MANEJAR EL CONOCIMIENTO Y EL APRENDIZAJE
}

THE INNOVATIVE ORGANIZATION'S GEMS: HOW TO DEAL WITH KNOWLEDGE AND LEARNING

\author{
Yolinel Hamel de Rodríguez \\ Isabel Pérez de Maldonado
}

UNIVERSIDAD PEDAGÓGICA EXPERIMENTAL LIBERTADOR 


\title{
LAS GEMAS DE LA ORGANIZACIÓN INNOVADORA: CÓMO MANEJAR EL CONOCIMIENTO Y EL APRENDIZAJE
}

\author{
THE INNOVATIVE ORGANIZATION'S GEMS: HOW TO DEAL WITH \\ KNOWLEDGE AND LEARNING \\ REVISIÓN DOCUMENTAL \\ Recibido:03-12-13 \\ Yolinel Hamel de Rodríguez* \\ Isabel Pérez de Maldonado** \\ UPEL \\ Aceptado: 28-03-14
}

\section{RESUMEN}

El presente artículo se inscribe en la modalidad documental y tiene como objetivo de reconocer las potencialidades de la Teoría General del Conocimiento y la Teoría de la Acción para potenciar las organizaciones innovadoras, cuyas particularidades las obligan a ser vanguardistas en el uso de tecnologías y a reconocer que el capital humano le aporta valor agregado cuando se le permite participar del entretejido organizacional. A partir de la revisión teórica referencial, se plantea la necesidad de generar una espiral del conocimiento, involucrando: al conocimiento tácito, que nace de lo aprendido en la cotidianidad y de las corazonadas; al conocimiento explícito, que se expresa a través del lenguaje formal en normas de procedimientos, manuales y reglamentos; a la teoría explícita, que se predica y a la teoría-enuso, que se practica. Se concluye que las organizaciones deben habilitar espacios armónicos que faciliten la exteriorización tanto de las capacidades, habilidades y destrezas individuales como de los supuestos, las inquietudes, las incertidumbres $\mathrm{y}$ hasta los descontentos.

Descriptores: Organizaciones innovadoras. Teoría General del Conocimiento. Teoría de la Acción.

\begin{abstract}
This documental article has as a purpose to recognize potentialities of the General and Action Theories to develop innovative organizations, whose characteristics oblige them to be modernist in the use of technologies and to recognize that human capital gives them appreciated value when allowed to participate in the organizational interfacing. From the theoretical review, it is proposed the need to generate a knowledge spiral, involving: explicit knowledge, expressed through formal language in procedure norms, manuals and regulations; to the explicit theory, which is predicated and to the in-use-theory, which is practiced. It is concluded that organizations should fit out harmonic spaces to facilitate the exteriorization of capacities, abilities and individual skills such as the suppositions, concerns, uncertainties and even discontents.
\end{abstract}

Keywords: Innovated organizations, General Theory of Knowledge, Action Theory

\footnotetext{
* Profesora de la UPEL-IPB. Maestría en Investigación Educacional. Diplomada en Comportamiento Organizacional y en Formación de Tutores de Trabajo de Grado y Tesis Doctorales. Doctoranda en Ciencias de la Educación VII Cohorte. Investigadora activa del NICO. yhamely@hotmail.com

**Doctora en Ciencias de la Educación. Investigadora adscrita al Núcleo de Investigación en Comportamiento Organizacional (NICO). isabelpdm@gmail.com
} 


\section{INTROITO}

"No es ninguna vergüenza aprender algo mientras lo ignoremos, es decir, mientras vivamos”

Séneca

Uno de los efectos de la sociedad posmoderna es la cantidad exorbitante y diversa de información, con una facilidad de acceso hasta ahora ilimitada. Este hecho definitivamente está cambiando nuestras vidas y a nuestras organizaciones, sobre todo a aquellas que quieren estar a la vanguardia, es decir, las innovadoras cuyo propósito es la creatividad, la novedad, la originalidad y la invención, tanto de productos como de servicios.

Lo anterior se escribe rápidamente, pero para lograrlo es preciso asumir conscientemente, entre otras cosas, que la información no decantada y utilizada, en muchos casos, resulta inútil. Por tanto, el reto de los gerentes, directivos y consultores es velar porque dentro de la organización se utilice eficaz y eficientemente la información y se logre su transformación en conocimiento existente, además de generar procesos en espiral que involucren la capacidad de aprender a aprender, de producir nuevos conocimientos y de ponerlos en acción.

Aquí, no se entiende al conocimiento como una cosa externa, sino como un recurso que está dentro de cada miembro de la organización y le aporta a ésta un valor agregado. Partimos de la premisa de que todos los procesos experienciales involucran al capital humano y generan algún tipo de conocimiento, consciente o inconsciente, necesariamente exteriorizable y, concomitantemente, algún aprendizaje que es preciso compartir. Por ello, este artículo de revisión documental persigue hacer un recorrido por la Teoría de la Generación del Conocimiento y la Teoría de la Acción, abordando: al conocimiento como capital intelectual o conocimiento activo, al proceso de espiral del conocimiento, al conocimiento tácito y al explícito, a las dimensiones del conocimiento: ontológica y epistemológica y a la generación del nuevo conocimiento.

Además se plantea: la construcción del aprendizaje, los niveles de aprendizaje, las dimensiones del aprendizaje organizacional: teoría explícita y teoría-en-uso y las técnicas para incrementar el aprendizaje organizacional. Finalmente, se reflexiona acerca de la organización creadora de conocimiento: creación y conversión del conocimiento, del espiral 
de creación, de los modelos para la creación de conocimiento, de la organización que aprende y del aprendizaje organizacional: de recorrido simple y de doble recorrido.

\section{GENERACIÓN DEL CONOCIMIENTO}

La Teoría de la Generación del Conocimiento, se fundamenta en la idea que las organizaciones deben ser innovadoras . Esto tiene varias significaciones: supone, en primer lugar, que la información proveniente del exterior para resolver problemas no sólo se procesa sino que se utiliza para crear nuevos conocimientos; y, en segundo lugar y más importante aún, que los conocimientos e informaciones procedentes del interior de la organización se utilizan para redefinir los problemas y las soluciones.

Ikujiro Nonaka e Hirotaka Takeuchi son los principales exponentes de esta teoría, quienes se encargaron de desmitificar la forma en que las compañías niponas han alcanzado el éxito, dado que por mucho tiempo se pensó erróneamente, tal como lo señalan los autores, que los japoneses se encargaban sólo de imitar y adaptar los productos sin innovar. Al realizar una investigación acerca de las características particulares de las empresas japonesas, estos autores elaboraron una teoría para que los occidentales pudieran comprender con detalle sus éxitos, al proporcionar una nueva explicación acerca de cómo algunas firmas japonesas usan el conocimiento para crear e innovar; además de desarrollar un modelo universal que revele la manera en que se debe dirigir una organización para alcanzar su mayor potencial.

El conocimiento, capital intelectual o conocimiento activo “....implica un conocimiento que pueda resultar de utilidad en la institución. Sin embargo, él no se convierte en capital hasta que se reconoce y comunica de modo que puede utilizarse o influir en beneficio de la institución...” (Martín Fernández, 2001, p. 177). Es decir, puede colectivizarse, a través de redes comunicacionales, informales y autoorganizadas que utilizan diversos medios: teléfonos, correo electrónico, foros on line, groupware o cualquier otro que les permita compartir lo que saben y resolver problemas que, en algunos casos, son generados por los propios miembros de la organización y que le imprimen sentido a ese nuevo conocimiento, que es, según Davenport y Prusak (2001), “...lo que hace funcionar a las organizaciones” (p.14). 
Su creación se constituye en un proceso en espiral, durante el cual dos tipos de conocimiento interactúan constantemente: el conocimiento tácito y el explícito (términos articulados por primera vez por el científico y filósofo húngaro Michael Polanyi). El primero de ellos se refiere al no codificado, al implícito, a la experiencia personal, a lo aprendido en el día a día, a las corazonadas, al entendimiento, a las ideas, a la intuición y a las habilidades; de allí que las creencias, los puntos de vista, las emociones, el instinto y los valores jueguen un papel fundamental en él. (Nonaka y Takeuchi, 1999; Wei Choo, 1999; Koulopoulos y Frappaolo, 2000; Martín Fernández, 2001; Davenport y Prusak, ob. cit; Gore, 2003; Ruiz, M. en Flores Fahara y Torres Herrera, 2010).

El conocimiento tácito “....contiene las llaves del reino del conocimiento corporativo. Es el banco de memoria más complejo, poderoso y mejor informado...” (Koulopoulos y Frappaolo, ob.cit., p. 164). Puede dividirse en dos dimensiones: la técnica y la cognoscitiva. La primera de ellas se refiere a las capacidades y habilidades no formales que hacen a una persona competente para llevar a cabo un oficio; mientras que la dimensión cognoscitiva comprende los modelos mentales, esquemas, creencias y percepciones de los individuos, que actúan constantemente para dar sentido a lo que se ve y se percibe, de manera que inciden en la forma de ver al mundo y a la realidad.

Por el contrario, el conocimiento explícito es el tangible, el codificado, el que todos ven, al que obedecen, el patente que se expresa a través del lenguaje formal en normas de procedimientos, manuales, documentos y reglamentos. (Nonaka y Takeuchi, ob. cit; Wei Choo, ob. cit; Koulopoulos y Frappaolo, ob. cit; Martín Fernández, ob. cit; Davenport y Prusak, ob. cit; Gore, ob. cit; Ruiz, M. en Flores Fahara y Torres Herrera, ob. cit); y en el caso concreto de la educación, a través, además de lo anterior, del currículo de los distintos niveles y modalidades del sistema. Para Nonaka y Takeuchi (ob.cit) el conocimiento explícito es el menos importante, mientras que el tácito es el que permite innovar, crear y generar.

Es fundamental comprender las diferencias entre el conocimiento tácito y el explícito, porque es lo que permitirá a los líderes, gerentes, directivos y docentes aplicar esta teoría en sus organizaciones. La clave de todo es entender que el conocimiento tácito, por su naturaleza individual, subjetiva, compleja e intuitiva, es de difícil procesamiento y transmisión en forma sistemática y lógica. Su transferencia precisa: a) “...un amplio 
contacto personal... y conversaciones exhaustivas...” (Davenport y Prusak, ob.cit., p. 109); b) la creación de "...una red de interacciones basada en patrones profundos e intangibles...” (Gore, ob.cit., p. 232); b) el uso de “...modelos sincrónicos de comunicación...” (Koulopoulos y Frappaolo, ob.cit., p. 32) y c) la rutina de crear “...ricos modos de conversación, entre ellos el empleo de analogías, metáforas o modelos, y de compartir anécdotas en la comunidad” (Wei Choo, ob.cit., p. 139).

En cambio, el conocimiento explícito es fácilmente divulgable, porque ha logrado ser sistematizado. Éste puede: a ) “...articularse... y transmitirse con facilidad entre los individuos...” (Koulopoulos y Frappaolo, ob.cit., p. 32); b) “...transmitirse utiliando un lenguaje formal y sistemático...” (Nonaka y Takeuchi, ob.cit., p. 65); y c) “...basarse en objetos o en reglas...” (Wei Choo, ob.cit., p. 133).

Ahora bien, tal como se mencionó ambos conocimientos son esenciales para que se produzca el nuevo conocimiento. Para lograrlo, los referentes sugieren varios procedimientos; por ejemplo, para Nonaka y Takeuchi (ob.cit.) la generación del nuevo conocimiento sucede cuando se logra que el tácito sea transmitido y diseminado en forma sistemática y lógica a toda la organización; es decir, se convierta en palabras, en ideas razonables que todos puedan comprender y que, por su naturaleza constructiva, todos puedan aprehender. Es precisamente en el proceso de conversión de tácito a explícito y viceversa cuando se produce el nuevo conocimiento.

Existen tres características que permiten convertir lo tácito en explícito: la primera es que se exprese lo inexpresable, mediante el uso del lenguaje figurativo, intuitivo, metafórico y simbólico; la segunda se refiere a la difusión o diseminación del conocimiento, a través del diálogo, la discusión, el intercambio de experiencias, el debate y la observación; y la tercera es la comprensión de la naturaleza compleja en que nace el nuevo conocimiento.

Koulopoulos y Frappaolo (ob.cit.) proponen a los gerentes relacionarse directamente con cada individuo y grupo para compartir conocimientos, a través de cuatro procesos a los que denomina intermediación, exteriorización, interiorización y cognición: a) la intermediación, se refiere a la conexión entre el conocimiento y la persona. Ésta puede ser asincrónica, cuando la exteriorización y la interiorización no son simultáneas, o sincrónica cuando sí lo son; b) la exteriorización, es la conexión del conocimiento con el 
conocimiento; para ello se debe capturar y almacenar en un lugar común, luego interpretarlo de forma que pueda ser útil y lograr clasificarlo y organizarlo en formatos que puedan ser utilizados fácilmente, lo cual supone una tarea titánica; c) la interiorización, conecta el conocimiento sistematizado con los usuarios, a través de buscadores especializados; y d) la cognición, en la que se vincula el conocimiento con el proceso para se produzca el metaconocimiento.

Wikström y Normann, citados por Wei Choo (ob.cit.), esquematizan tres procesos para la creación del nuevo conocimiento: a) los generativos, que crean el nuevo conocimiento; b) los productivos, que lo operacionalizan y c) los representativos que lo difunden y transfieren. Por su parte, Leonard-Barton propone cuatro actividades de formación del conocimiento: a) la resolución compartida de problemas; b) la experimentación y creación de prototipos; c) la puesta en práctica para integrar los nuevos procesos y herramientas y d) la importación del conocimiento. (Ibidem).

\section{Dimensiones de la Teoría de la Creación del Conocimiento Organizacional}

Dimensión Ontológica: parte del principio de que el conocimiento es creado por los individuos. En este sentido, Nonaka y Takeuchi (ob.cit.) señalan que la creación del conocimiento dentro de la organización debe ser entendida como un “...proceso que amplifica organizacionalmente el conocimiento creado por los individuos y lo solidifica como parte de la red de conocimiento de la organización...” (p. 65); el cual debe abarcar cada vez a más miembros, convirtiéndolos en una creciente comunidad de interacción, que se trasladan entre fronteras intra e interorganizacionales.

Dimensión Epistemológica: para explicar esta dimensión, Nonaka y Takeuchi (ob.cit.) citan las diferencias, que establece Michael Polanyi, entre el conocimiento tácito y el explícito. A este respecto señalan que el primero “...es personal y de contexto específico y, así, difícil de formalizar y comunicar...” (p. 65). Influyen en él los modelos mentales, los cuales son, según lo afirmó Jonson-Laird citado por los autores, “...esquemas, paradigmas, perspectivas, creencias y puntos de vista que ayudan a los individuos a percibir y definir su mundo...” (p. 66). Mientras que el conocimiento explícito o codificado, tal como se ha venido señalando, es el que puede transmitirse mediante el uso del lenguaje formal y 
sistemático. Entonces, este autor sostiene que los seres humanos adquieren el conocimiento al crear y organizar las nuevas experiencias. Por lo tanto, la naturaleza epistemológica de esta teoría se basa en el principio de que los individuos crean el conocimiento involucrándose con el objeto.

\section{Construcción del Aprendizaje desde el conocimiento organizacional}

En la sección anterior abordamos la generación del conocimiento como un proceso que permite a los individuos aumentar su creatividad y su potencial de innovación. Ahora, se propone la observación del potencial cognitivo del individuo como una aptitud hacia el aprendizaje, entendido como “...un proceso de expansión de la capacidad de acción efectiva tanto en los individuos como en las organizaciones...” (Autor, 2001, p. 65), que “...tiende a sufrir los efectos de la brecha entre la idea y la acción...” (Perkins en Gore, ob.cit. p. 14).

En tanto que, la aptitud hacia el aprendizaje, se divide en las aptitudes para la reflexión y para la indagación. Según Senge (1992), las primeras “...se relacionan con desacelerar nuestros procesos de pensamiento para cobrar mayor conciencia de cómo formamos nuestros modelos mentales y cómo éstos influyen sobre nuestros actos” (p. 244), mientras que las aptitudes para la indagación “...conciernen a nuestro modo de operar en interacciones directas con los demás, sobre todo cuando abordamos temas complejos y conflictivos" (ibidem).

Sin embargo, Argyris, en Harvard Business Review (2000), plantea que las personas no saben cómo aprender, lo cual dificulta el éxito de la organización en un ambiente que cada día se perfila más complejo, sobre todo para aquellas que quieren ser innovadoras. La situación empeora porque la mayoría de las instituciones no están conscientes de esta problemática, lo cual supone la inexistencia de herramientas para resolverla. El motivo, señala el autor, es que “...entienden mal lo que es el aprendizaje y cómo originarlo...” (p. 92). Ya que un número importante de personas definen el aprendizaje de un modo simplista, “...como una mera resolución de problemas...” (p. 93) del entorno. Una manera de solventar este dilema es que dentro de la organización se analicen las dificultades con una visión crítica de su propio comportamiento, cultura y clima, lo cual contribuirá a resolver la fuente del problema, cambiando su modo de actuar y aprendiendo en el proceso. 
Para lograrlo, proponemos la aplicación de la Teoría de la Acción, la cual aparece por primera vez en la obra Organizational Learning: A Theory of Action Perspective, escrita por Chris Argyris y Donald Schön en el año 1978. Allí, se plantea a profundidad la relación entre el pensamiento y la acción, tal como lo señala Picón Medina (1994), “es una teoría que concibe al hombre como un ser que diseña sus acciones, las ejecuta y evalúa sus consecuencias; adopta sus diseños cuando las consecuencias responden a sus intenciones y las modifica... cuando los resultados les son adversos” (p. 48). Esta teoría contempla un acercamiento metodológico, teórico y de investigación, que puede ser utilizado en forma individual, grupal y organizacional.

Un acercamiento metodológico porque proporciona técnicas para intervenir en sistemas complejos y diagnosticar las causas originarias de los comportamientos que, de alguna manera, producen resultados poco deseados; teórico, al proveer una matriz de trabajo, que coadyuva a entender el comportamiento organizacional y la cultura, con el objeto de generar estrategias promotoras de cambios basados en el aprendizaje; e investigativo, porque proporciona herramientas para explorar acciones individuales, grupales y organizacionales.

La conducta de los individuos, según esta teoría, está regida tanto por los significados como por sus intenciones, permitiéndole a éstos dejar de ser actores y asumir acciones posibilitadoras de dar significados al ambiente que les rodea; es decir, comportarse como verdaderos autores, ya que, según Argyris y Schön citados por Picón Medina (ob.cit), la "conducta humana intencional y el aprendizaje humano no requieren ser explicados en términos de reforzamientos o extinciones de los patrones de conducta... sino en términos de un proceso de construcción, prueba y reconstrucción de cierto tipo de conocimientos...” (p. 51). Es por ello que este autor considera la fundamentación epistemológica de la conducta y del aprendizaje humano no desde un punto de vista conductista: estímulo, respuesta, refuerzo; sino desde el enfoque constructivista.

Otro aspecto fundamental está relacionado con los niveles de aprendizaje. Éstos son: 


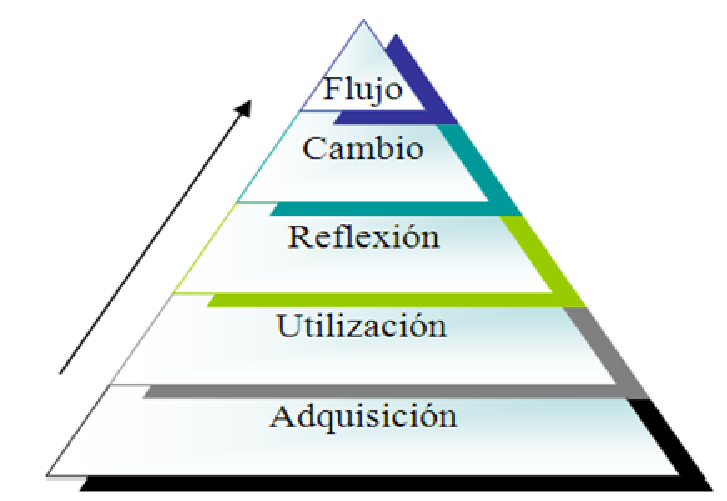

Gráfico 1. Niveles de aprendizaje organizacional. Adaptado de Guns (1996, pp. 17-18)

En el primer nivel, de adquisición, los miembros desarrollan actitudes, creencias, valores y principios y obtienen información, conocimiento y habilidades y destrezas para desempeñar un oficio, incluso antes de ser contratado por una empresa. El segundo nivel, de utilización, se alcanza cuando los miembros usan los conocimientos adquiridos. Ésta puede llegar a ser sólo una actividad, entonces no se considera un aprendizaje real, o puede estar acompañada de una espiral de retroalimentación, que vincule la experiencia vivida (lo logrado) con la meta original (lo que se pretendía alcanzar). En el tercer nivel, de reflexión, los miembros de la organización piensan analíticamente en lo que hacen, desde una perspectiva más amplia, que incluya cuestionamientos y la superación de suposiciones y prejuicios.

En el cuarto nivel, de cambio, se combina el pensamiento y la acción. Se responde a una situación mediante una estrategia que permita generar aprendizajes. En el último nivel, de flujo, el aprendizaje y la acción fluyen; se combinan los aprendizajes más elementales y se refuerzan unos a otros sin esfuerzo consciente. Es parecido, metafóricamente, a la confluencia de dos ríos, uno de ellos es el aprendizaje y el otro la actividad. Cuando se unen no se sabe cuál es cuál. Según Fullan, citado por Ruiz en Flores Fahara y Torres Herrera, 2010, “...el aprendizaje es un cambio que eventualmente se vuelve permanente; cuando algo nuevo deja de ser nuevo para ser algo cotidiano, la organización hace suyo este nuevo conocimiento a través de la acción...” (p. 50). Y lo más importante es que en el nivel flujo, esto sucede de manera natural. 


\section{Dimensiones del Aprendizaje Organizacional}

La Teoría de la Acción distingue dos dimensiones: la teoría explícita, expuesta o adoptada y la teoría-en-uso o tácita. La primera es la que se “...predica, conformada por los fines, los supuestos y los valores que según lo que la persona dice, guían su conducta...” (Argyris y Schön, citados por Picón Medina, ob.cit., p. 54). Para Wey Choo (ob.cit.), la teoría explícita o teoría adoptada de la organización, es aquella que se quiere “...proyectar hacia el mundo externo y hacia sus miembros...” (p. 261). Un ejemplo de ella son, según este autor, "un examen de los documentos formales de la organización, como sus organigramas, declaraciones de planes de acción, descripciones de tareas y archivos...” (ibidem).

En cambio, la teoría-en-uso es la que se “...practica, conformada por los supuestos implícitos que en realidad guían la conducta observada” (Argyris y Schön, citados por Picón Medina, ob.cit., p. 54). Para Wey Choo (ob.cit.), la teoría-en-uso es tácita y se exterioriza o manifiesta en las acciones y conductas, tanto de los miembros de la organización, de manera individual, como de la organización misma; ésta es “...informal por naturaleza...tácita y con frecuencia no codificable; por lo mismo, está relacionada con las representaciones, el conocimiento tácito y las heurísticas y los atajos que vimos que emplean las organizaciones cuando perciben, construyen conocimiento y toman decisiones...” (p. 264). Entonces, esta teoría también está determinada por las convenciones sociales, la cultura y los modelos mentales, debido a que en ella intervienen todos los procesos generativos de la conducta del individuo: cognoscitivos, socioafectivos, históricos y ético-morales.

Estas teorías "pueden ser, y generalmente son, diferentes y hasta opuestas entre sí, con el agravante de que sólo en muy contadas ocasiones el actor se percata de esta incongruencia” (Méndez, 2000, p. 47); también para Wey Choo (ob.cit.) la teoría-en-uso es incompatible con la teoría explícita, es decir, existe una incoherencia entre lo que se predica y lo que se practica, de la cual se puede estar consciente o no. Por ejemplo, un profesor puede decir (teoría explícita) que incentiva la formación del pensamiento crítico de sus estudiantes, pero cuando alguno de ellos lo increpa con argumentos, hace caso omiso (teoría-en-uso). Según Senge, (ob.cit.) la brecha existente entre ambas teorías pueden causar desaliento, ya que se puede pensar que son actos cínicos; sin embargo, no es 
necesariamente ese el motivo, algunas veces responde a nuestra percepción, a nuestros modelos mentales e incluso a las convenciones sociales, no a la hipocresía. Esto tiene que ver con la complejidad del ser humano y de la sociedad.

Afortunadamente, estas teorías pueden ser modificadas, cuando los individuos, según Argyris y Schön, citados por Wey Choo (ob.cit.)

Actúan sobre la base de sus imágenes y sobre sus mapas compartidos, con expectativas de resultados según ciertos patrones, que a su experiencia subsiguiente confirma o no confirma. Cuando hay un desajuste entre resultado y experiencia (error), los miembros pueden reaccionar al modificar sus imágenes, mapas y actividades para así volver a armonizar las expectativas y los resultados” (p. 262).

Para lo cual debe existir un proceso de reflexión sobre la acción y sobre los modelos mentales. Se debe estar atento de lo que hacemos y lo que pensamos al respecto. Al final, se espera que el individuo sea y actúe (soy-en-acción), siempre que se pueda, de manera armónica y no disonante. Aquí, entra en juego lo que Senge (ob.cit.) llama "reflexión para la acción”, que es la capacidad para reflejar el pensamiento mientras se actúa; también para Schön, citado por Senge (ib.), la reflexión sobre el pensamiento y la acción es lo que hacen los individuos para aprender mientras realizan las cosas, de allí que existan frases como: pensar bien, aprender a aprender y aprender haciendo. Lógicamente, estas actitudes para la reflexión requieren que el individuo contraste la realidad con la interpretación, que es lo que se conoce como brincos de abstracción, los cuales ocurren para Senge (ob.cit.), “...cuando pasamos de las observaciones directas (datos concretos) a generalizaciones no verificadas. Estos brincos obstaculizan el aprendizaje porque se vuelven axiomáticos. La mera suposición se transforma en hecho” (p. 246).

La localización de los brincos de abstracción es la primera técnica de la ciencia de la acción, para lo cual es preciso preguntarse cuáles son los datos sobre los cuales se basa esta generalización; es decir, se ve la realidad desde un punto de vista objetivo, sin muchas conjeturas ni interpretaciones basadas en la propia creencia y no en los hechos reales. Luego, hay que preguntarse si se está dispuesto a considerar que la generalización puede ser inexacta o equívoca, es decir, si se está en capacidad de cuestionar su propia aseveración. "Si la respuesta es negativa no tiene caso continuar” (Senge, ob.cit., p. 248). 
La segunda técnica de la ciencia de la acción es llamada la columna izquierda, la cual consiste en observar cómo operan los modelos mentales del individuo en determinadas circunstancias, conllevando a un proceso de reflexión sobre su influencia en la manera de percibir el mundo y de actuar. Esta técnica comienza con "la relación de una situación específica donde estoy interactuando con una o varias personas de una manera que no funciona: no produce aprendizaje ni avanza” (Senge, ob.cit., p. 249). Luego, se reproduce la conversación por escrito, con forma de guión, en el lado derecho de la página, mientras que en el izquierdo se escribe lo que se piensa pero se calla en cada etapa de la conversación.

Esta técnica sirve para develar los supuestos ocultos y su influencia sobre la conducta; además de detectar las oportunidades frustradas para el aprendizaje en situaciones de conflicto. Sin duda, una vez más se trata de contrastar la perspectiva con los datos reales, al contribuir a aclarar los supuestos y los ocultamientos que perjudican las relaciones entre los individuos y se convierten en barreras de comunicación, permitiendo que emerjan los intríngulis detrás de cada posición.

Una tercera técnica es conocida como equilibrio entre la indagación y la persuasión, para generar la co-construcción del aprendizaje: el aprendizaje cooperativo, colaborativo o en equipo. La ciencia de la acción nos dice que es tan importante persuadir como indagar, es por ello que debe existir un equilibrio entre ambas. De hecho, "la persuasión sin indagación genera más persuasión y crea un arquetipo sistemático... que se llama escalada” (Senge, ob.cit., p. 252), la cual explica así: “cuanta mayor vehemencia exhiba A, más amenaza a B. Entonces, B argumenta con mayor fiereza, y así sucesivamente” (ibidem). Este efecto, se detiene cuando hay un aprendizaje productivo; es decir, cuando se combina la indagación con la persuasión, a lo que se denomina indagación recíproca, la cual se logra al someter a un examen público su pensamiento, argumentando bien sus ideas. Ello conduce a la necesidad de clarificar los hechos y exponer los datos de tal manera que posibiliten encontrar los defectos de su propio punto de vista.

Se puede desarrollar por etapas: la primera consiste en indagar las perspectivas de otras personas con las que no se está de acuerdo, lo cual se puede lograr al solicitarle a la persona que explique cómo llegó a ese punto de vista, o que describa con mayor detalle la situación o la conclusión. Posteriormente, en una segunda etapa, se exponen las propias 
opiniones, pero invitando a los demás a indagar también, lo cual permite descubrir nuevas perspectivas.

Sin duda, al hacer uso de las técnicas anteriores, cada individuo dentro de la organización debe orientar su aprendizaje para obtener cambios en su acción. Para ello, es necesario descubrir la brecha que existe entre "las teorías que guían (lo que decimos) y muestras teorías-en-uso (las teorías que justifican nuestros actos)” (Senge, ob.cit., p. 256). Este autor plantea que de no ser así, se puede caer en el error de creer que se ha aprendido algo "sólo porque adoptamos un lenguaje o conceptos nuevos, aunque nuestra conducta no se haya modificado" (ibidem).

\section{Organización Creadora de Conocimiento}

La organización creadora de conocimiento, según Nonaka, en Harvard Business Review (2000), está centrada tanto en las ideas como en los ideales de sus miembros, quienes fomentan la innovación. Este autor concibe a la organización no como una máquina, sino como un organismo viviente, porque puede tener un sentido colectivo de identidad y de finalidad última; “...el equivalente al autoconocimiento de la persona -una idea compartida acerca de lo que la empresa significa, de hacia dónde se dirige, de la clase de mundo en que se desea vivir, y... de cómo hacer realidad ese mundo-” (p. 27). Por ello, es importante que los miembros de la organización recreen el mundo siguiendo su visión y su misión, para que se produzca la innovación, ya que “...crear un nuevo conocimiento significa literalmente recrear la empresa y a cada una de las personas que trabajan en ella mediante un proceso ininterrumpido de autorrenovación personal y empresarial” (p. 28).

Para lograrlo, lo primero que se debe tener en cuenta es que el conocimiento tácito y el explícito no son entidades separadas, sino complementarias. Por lo tanto, la fundamentación de esta teoría, en cuanto a la creación del conocimiento, es el principio de que éste se construye mediante la interacción social y permanente de ambos tipos de conocimiento; es decir, del tácito y del explícito. A tal interacción es a la que los referentes llaman conversión del conocimiento, la cual se puede producir mediante cuatro formas: 


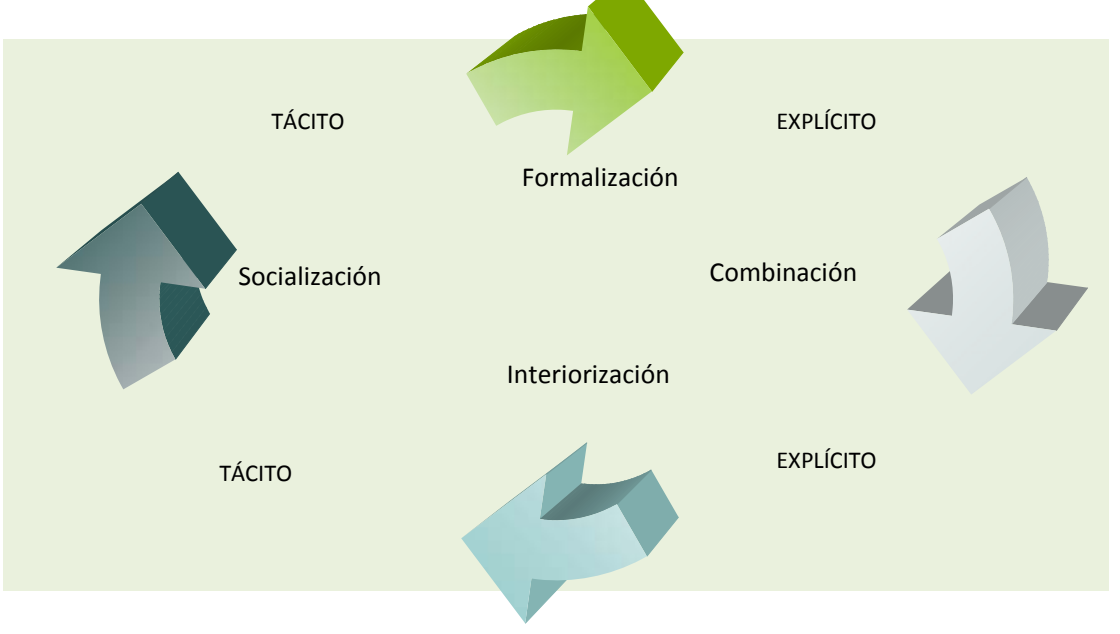

Gráfico 2. Procesos de transformación y creación de conocimiento. Tomado de Bolívar (2000,p.157)

La primera conversión se produce de tácito a tácito y se le llama socialización, debido a que es un proceso que consiste en compartir experiencias. (Nonaka y Takeuchi, 1999; Wei Choo, 1999; Nonaka, en Harvard Business Review, 2000; Bolívar, 2000; Martín Fernández, 2001). El sólo hecho de escuchar a otros, sin hacer uso del lenguaje propio, puede hacer que un individuo aprenda. Un hombre puede aprender mecánica observando, imitando y practicando con un mecánico, al igual que un alumno puede aplicar una fórmula matemática para resolver un problema, o un bebé repetir un sonido que su mamá le expresa. La clave para obtener un conocimiento tácito es la experiencia compartida.

En la organización, la socialización generalmente comienza con la creación de un campo de interacción, en el que sus miembros comparten sus experiencias y modelos mentales. Este proceso de socialización genera un conocimiento armonizado. Para explicar esta forma de conversión Wei Choo (ob.cit.) hace referencia el trabajo de Lave y Wenger, quienes proponen que “...los aprendices observan y aprenden a través de una legítima participación periférica...” (p. 144), como observadores participantes que deciden el momento de abandonar al maestro o de regresar a él.

La segunda conversión se llama exteriorización o formalización y se origina de tácito a explícito, ya que es un proceso a través del cual se declara el conocimiento tácito en forma de conceptos explícitos, asumiendo formas de definiciones, conceptos, metáforas, hipótesis, analogías, modelos o anécdotas, lo que facilita el intercambio de conocimiento 
con otras personas. (Nonaka y Takeuchi, 1999; Wei Choo, 1999; Nonaka, en Harvard Business Review, 2000; Bolívar, 2000; Martín Fernández, 2001). Un ejemplo de ello es cuando el bebé utiliza las palabras y sonidos con fines determinados: pedir agua porque tiene sed, o tetero porque tiene hambre; o un alumno comprende que al aplicar el algoritmo de la suma aumentan las cantidades.

En la organización, la exteriorización empieza a partir de un diálogo o reflexión colectiva significativos, en los que el uso de una metáfora o una analogía apropiada ayudan a los miembros a enunciar el conocimiento tácito oculto, que de otra manera resulta difícil enunciar. La exteriorización genera conocimiento conceptual. (Nonaka y Takeuchi, ob.cit., p. 81).

La tercera conversión se desencadena de explícito a explícito y se llama combinación. Es un proceso en el que se sistematizan los conceptos para generar un sistema de conocimiento, que implica la combinación de distintos cuerpos de conocimiento explícito (Nonaka y Takeuchi, 1999; Wei Choo, 1999; Nonaka, en Harvard Business Review, 2000; Bolívar, 2000; Martín Fernández, 2001).

De modo que los individuos intercambian y combinan conocimiento a través de distintos medios, tales como documentos, reuniones, juntas, focus group, conversaciones telefónicas, correos electrónicos, foros on line, entre otros. El procesamiento de la información existente, la cual se genera tras un proceso de organización, clasificación, combinación y categorización del conocimiento explícito, puede llegar a producir un nuevo conocimiento. Por ejemplo, cuando un estudiante utiliza el parafraseo, analiza un texto o estudia y presenta un examen está generando una combinación de conocimientos. La combinación origina conocimiento sistémico. Éste es el conocimiento más frecuentemente producido en la educación formal.

La última conversión se denomina interiorización y se produce de explícito a tácito; está relacionada con el aprender haciendo. “...Cuando las experiencias son internalizadas en la base del conocimiento tácito de los individuos a través de la socialización, la exteriorización y la combinación, en la forma de modelos mentales compartidos y del conocimiento tácito técnico, se vuelven activos muy valiosos...” (Nonaka y Takeuchi, ob.cit., p. 78) 
Pero, para que se produzca el conocimiento organizacional, que por definición es el que “...se refiere al conjunto de saberes y prácticas que, inscriptos en la memoria de la organización, conforman su cultura y su modo particular de pensar, sentir y hacer...” (Romero, 2004, p. 129), se precisa que el conocimiento tácito que los miembros acumulan de forma individual se socialice con sus pares, superiores o colaboradores, así se construye una nueva espiral de creación del conocimiento. Para ayudar a que el explícito se vuelva tácito, se debe verbalizar; es decir, expresar con palabras, lo cual se logra al realizar mandalas, mapas mentales, ideogramas, historias orales, diagramas, flujogramas, documentos y manuales de procedimiento, cargados de su propia percepción. La interiorización crea conocimiento operacional.

Todos ellos interactúan entre sí en la espiral de creación del conocimiento. En este sentido Wei Choo (ob.cit.) señala que “...la organización tiene que proporcionar el combustible y el medio ambiente para que la chispa creativa o energía para la innovación se prenda y alimentar la llama hasta convertirla en algo que la organización pueda utilizar...” (p. 143). Así, se espera que ésta provea los contextos apropiados para facilitar la exteriorización del conocimiento, el cual siempre se inicia como un acto individual, “...podemos saber mucho más que lo que podemos expresar...” (Michael Polanyi, citado por Nonaka en Harvard Business Review, ob.ci., p. 30).

Lo anterior se puede lograr a través de la interacción de los miembros de la organización, promoviendo actividades grupales, conversatorios, reuniones informales y redes comunicacionales, a fin de que se genere la creatividad, la innovación y la acumulación de conocimiento, tanto a nivel individual como grupal y organizacional.

\section{Modelos de la Creación del Conocimiento}

\section{a) Según Nonaka y Takeuchi}

El modelo integral de creación del conocimiento organizacional consta de cinco fases: la primera fase consiste en compartir el conocimiento tácito. Para estos autores, el conocimiento tácito es una fuente inmaculada y abundante de nuevo conocimiento, pero debe recordarse que éste es producto de la experiencia personal, y, por lo tanto, de difícil comunicación. Para que esto suceda, resulta interesante que la organización invite a sus miembros y constantemente provea lugares, espacios y tiempos donde los individuos 
puedan interactuar mediante diálogos cara a cara para compartir sus experiencias y trabajar juntos, a fin de alcanzar metas comunes.

La segunda fase se basa en la creación de conceptos. En la fase anterior se propició la construcción de modelos mentales compartidos, que se exteriorizan en la segunda fase a través del diálogo continuo (mediante palabras y frases) que posibilitan la cristalización de conceptos explícitos.

La tercera fase se fundamenta en la justificación de los conceptos. Es necesario asegurarse de que los conceptos creados en las etapas previas sean verdaderamente válidos para la organización y para la sociedad. En un proceso de decantación, los individuos se aseguran de que los conceptos generados cubren las necesidades y las expectativas de la organización, y ésta se encarga de guiar el proceso y de asegurarse de que efectivamente esos conceptos construidos son útiles para la sociedad y satisfacen sus necesidades.

La cuarta fase consiste en construir un arquetipo. Esa justificación que se produjo en la fase anterior se convierte en un arquetipo, es decir, en algo tangible y concreto. Por ejemplo, un prototipo, un modelo o una maqueta.

La quinta fase se basa en expandir el conocimiento. La creación del conocimiento organizacional “...es un proceso interminable que se actualiza a sí mismo constantemente” (Nonaka y Takeuchi, ob.cit., p. 101). El nuevo concepto que se creó, se justificó y se modeló o concretó sigue un camino interminable de ciclos creativos de conocimiento a otros niveles ontológicos distintos.

\section{b) Según Collison y Parcell}

Proponen un modelo holístico que relaciona “...los procesos de aprendizaje y la captura y transferencia del conocimiento con las actividades cotidianas..." (Collison y Parcell, 2003, p. 46). La primera fase de su modelo consiste en asegurarse de aprender antes, durante y después; la segunda, se apoya en el uso del conocimiento capturado, de manera que pueda ser reutilizado. Estas fases se consiguen con la colaboración de las redes de trabajo y las comunidades y reflexionando en torno a los objetivos organizacionales y a los resultados obtenidos. 


\section{c) Según Toshiba}

Toshiba desarrolló un modelo denominado Knowledge Works, que se fundamenta en las 3K, esto es Knach, consiste en la facilidad de hacer algo; Know-How, es decir el conocimiento procedimental que responde al cómo hacer las cosas de forma eficiente y el Knowledge, que es la consciencia de las cosas que se hacen, es decir, la metacomprensión de los procesos. (Martín Fernández, ob.cit.)

\section{d) El cuestionario KMAT}

Es un modelo de instrumento que realizó la American Productivity \& Quiality Center con el fin de aproximarse al concepto de organizaciones inteligentes. En él se analizan: las propiedades del liderazgo, las prácticas culturales de las organizaciones que gestionan su conocimiento, las propiedades de la tecnología del conocimiento, los resultados y las propiedades de los procesos del conocimiento. (Martín Fernández, ob.cit.)

\section{e) Según Rojas Quiñones}

Propone un modelo de gestión del conocimiento que comprende dos momentos simultáneos e inseparables uno de otro. El primero se basa en la generación de conocimiento “....ya sea en términos de creación pura, de reelaboración y actualización del conocimiento ya poseído por la institución, o de su obtención sistematizada y adaptación de fuentes externas, usualmente conocidas como investigación...” (Rojas Quiñones, 2006, p. 51). El segundo momento, íntimamente ligado al anterior, consiste en ponerlo en uso, lo cual se puede lograr incorporando canales adecuados de divulgación de manera que permee a toda la organización y la proyecte como una institución innovadora que asuma los nuevos retos de la sociedad del conocimiento.

La organización cumple con los mismos ciclos del organismo humano: nace, crece, eventualmente se reproduce y finalmente muere. Sin embargo, a diferencia de los individuos, esto último puede aplazarse en las organizaciones al irlas renovando día a día. Cuando esto sucede, la organización es conocida como una de aprendizaje; es decir, una organización capacitada para adaptarse a los cambios del entorno, mediante una renovación permanente tanto de sus estructuras, como de sus prácticas. Con ello se comprometen, según Koonz y Weihrich, citados por el autor, a la “...sistemática solución de problemas, la 
experimentación y la búsqueda permanente de nuevos conocimientos... y a la tolerancia al fracaso...” (p. 235).

\section{La Organización que Aprende}

Una organización que aprende, es un sistema (Ruiz en Flores Fahara y Torres Herrera, 2010; Rojas Quiñones, 2006; Davenport y Prusak, 2001), que se adapta a sus propios requerimientos y a los de la sociedad, generando aprendizajes nuevos derivados de la acción de sus miembros, organizados en equipos, para resolver sus problemas. Koulopoulos y Frappaolo (2000), refieren que “... su objetivo es mejorar los niveles de innovación a través de toda la organización” (p. 49). Aunque, tal como lo señala Guns (1996), “...todas las organizaciones aprenden, pero no todas se basan en el aprendizaje... Las organizaciones basadas en el aprendizaje se enfocan en que el trabajo se realice mejor. Consideran el aprendizaje como la forma idónea de mejorar a largo plazo el rendimiento" (p. 2).

Son organizaciones que están más acordes con la naturaleza humana (O’Brien en Senge, ob.cit.). Que reconocen y corrigen los errores, aunque eso signifique flexibilizar a la organización, de tal manera que emerjan nuevos aprendizajes, nuevos procedimientos y nuevas respuestas derivadas de los nuevos desafíos (Aguerrondo, 1996). Que aprenden a aprender, comenzando por reconocer y examinar sus propios problemas, a fin de obtener lecciones de ellos; se hacen competentes y se mantienen así (Swieringa y Wierdsma, 1995). Que son expertas en crear, adquirir y transmitir conocimiento; además, modifican su conducta a fin de adaptarse a las nuevas ideas y conocimiento (Garvin en Harvard Business Review, 2000) y que se someten continuamente a la prueba de la experiencia y la transforman en conocimiento accesible y pertinente a su misión. (Senge, Roberts, Ross, Smith y Kleiner, 2004).

El aprendizaje organizacional puede producirse en el momento en que los miembros de la organización detectan errores y actúan para corregirlos, bien sea modificando sus estrategias, los supuestos o las normas. Éste puede desencadenarse de dos modos: el primero, conocido como Aprendizaje de Recorrido Simple (Picón Medina, 1994) o Aprendizaje de Circuito Sencillo (Wey Choo, 1999). Se origina cuando el error es solucionado sin modificar las teorías-en-uso, es decir, se genera una realimentación entre 
los resultados detectados y la acción ejercida sin cuestionar las normas existentes. De esa manera, se aumenta la eficacia de la organización manteniendo sus objetivos inalterables, lo cual constituye el propósito fundamental de este tipo de aprendizaje. Es ventajoso cuando sólo se requiere una adaptación para solucionar un problema.

Es conveniente advertir que cuando los gerentes controlan unilateralmente las metas, el ambiente, las tareas, la situación, la información y las decisiones, pueden emerger conductas defensivas en los miembros de la organización, lo cual entorpece el aprendizaje organizacional, generando sólo un Aprendizaje de Recorrido Simple (Picón Medina, 1994; Méndez, 2000) o Modelo I (Bolívar, 2000), el cual está constituido por cuatro directrices: a) lograr los propósitos tal como han sido definidos; b) intención de ganar siempre y de siempre tener la razón; c) eliminación de sentimientos negativos y d) prevalencia de lo racional.

Sin embargo, existen ocasiones en que el aprendizaje requiere ser generativo, que es el segundo modo en que puede producirse el aprendizaje organizacional. Éste suele estar acompañado del primero “...cuando se presenta una brecha en el entendimiento.... que implica volver a pensar y rediseñar modelos mentales, y cambiar rutas mediante las cuales las personas se adaptan, para moverse de donde están hacia donde aspiran estar...” (Koulopoulos y Frappaolo, 2000, p. 180); de manera que surja una modificación de las normas de trabajo existentes para poder corregir el error, “...que a su vez demanda una reestructuración de estrategias y supuestos relacionados con las normas...” (p. 263).

Desencadenándose el Aprendizaje de Doble Circuito (Wey Choo, 1999) o Aprendizaje de Doble Recorrido (Picón Medina, 1994) o Modelo II (Bolívar, 2000), en el que predomina una comunicación abierta y una realimentación vinculada con la localización del error tanto con las acciones generadas por los miembros de la organización como con las normas existentes; de manera que al resolver los problemas ocasionados por las normas incompatibles o perturbadoras $\mathrm{y}$ emprender acciones encaminadas a reestructurarlas o reelaborarlas, considerando los nuevos escenarios, se estará en vías de potenciar el desarrollo y evolución de la organización.

También, cuando se utiliza una información públicamente validada, lo cual promueve la toma de decisiones libres y un compromiso compartido por los miembros de la organización, se genera un aprendizaje de este tipo; debido a que se logra que quienes 
tengan competencias pertinentes compartan el control de las acciones, minimizando las situaciones de conflicto y, por ende, las conductas defensivas. Todo ello se traduce en una organización más eficiente que está en vías de transformarse en una organización innovadora.

\section{REFLEXIÓN FINAL}

Cuando se quiere transformar una organización en función de las demandas sociales y de las propias, es preciso, entre otras cosas, generar conocimiento; es decir, aumentar la capacidad de respuesta de los miembros, tener mayores argumentos para la toma de decisiones, conectarse con las necesidades del entorno, incorporar procesos innovadores y aprender en todo el proceso, utilizando tanto el conocimiento explícito, es decir el declarado, codificado, sistematizado en documentos, reglamentos y manuales de procedimiento; como el implícito, que se origina a partir de los sentimientos, las habilidades, las experiencias y la intuición. La situación deseada por los miembros de la organización es que ambos se asocien, se combinen y se aprehendan con el fin de alcanzar la creatividad y la innovación.

Por tanto, la creación del conocimiento tiene que ver tanto con los ideales como con las ideas; es por ello que se afirma que cuando se quiere innovar es preciso que todos los miembros de la organización se aboquen a la tarea de la renovación personal y organizacional, para construir el nuevo conocimiento. No basta con que un investigador, un interventor, el director de una escuela o un grupo de docentes quieran cambiarla, sino que todos los miembros compartan el mismo ideal y trabajen en armonía y con la misma fuerza.

Específicamente aplicado al ámbito educativo, se asegura que el principal capital de las organizaciones educativas universitarias es el intelectual, por lo tanto, sus fuentes se encuentran tanto en el cuerpo rectoral, docente, administrativo y de servicio como en los estudiantes, es decir, en el capital humano, el cual se define en función: de los objetivos, de la misión de la organización, de sus metas, en fin, de lo que se desea alcanzar.

Sin embargo, para que las habilidades y el conocimiento puedan hacer aportes significativos, deben cumplir ciertos requisitos, tales como: estar atentos a las posibles fuentes de problemas o dificultades, lo cual permitirá crear conocimientos; además, compartir el conocimiento con el resto de los miembros y generar el aprendizaje tan 
deseado en la organización, ya sea convirtiendo el conocimiento explícito en tácito, a lo que denominan recolección; clasificando, almacenando y recuperando el conocimiento, a lo que llaman organización y facilitando el acceso a la información, conocido como obtención. Una vez creado el conocimiento, es imprescindible aplicarlo, es decir usarlo para el bien común; sin embargo, es conveniente aclarar que,

La conformación de organizaciones que aprenden no debe verse como una panacea, sino como un estilo de vida organizacional. El aprendizaje está ligado a los seres humanos desde su nacimiento, es una capacidad innata del individuo; por tanto, si las organizaciones están conformadas por individuos, es de esperarse que juntos aprendan, es decir, que la organización aprenda (Autor, 2010, p. 11).

Finalmente, los referentes coinciden en manifestar que si se persigue la creación o refundación de las organizaciones en creadoras de conocimiento y que aprendan en el proceso, es vital habilitar espacios armónicos para facilitar la exteriorización tanto de las capacidades, habilidades y destrezas individuales como de los supuestos, las inquietudes, las incertidumbres y hasta los descontentos. Ya que los miembros de la organización la comprenden de acuerdo a su propia actuación a través de las palabras, utilizadas para “...lograr acciones, y el resultado de esas acciones les va permitiendo entender lo que sucede” (Weick, citado por Gore, ob.cit., p. 319).

Para conseguirlo se deben promover los focus group, conversatorios, reuniones informales, dinámicas grupales y redes comunicacionales, a objeto de que emerjan las ideas, la creatividad, la innovación y la acumulación de conocimiento en acción, tanto en el nivel individual como en el grupal y en el organizacional.

\section{REFERENCIAS}

Aguerrondo, I. (1996). La escuela como organización inteligente. Buenos Aires: Troquel.

Bolívar, A. (2000). Los centros educativos como organizaciones que aprenden. Madrid: La Muralla.

Collison, C. y Parcell, G. (2003). La gestión del conocimiento. Barcelona, España: Paidós.

Davenport, T. y Prusak, L. (2001). Conocimiento en acción. Buenos Aires: Pearson Education. 
Flores Fahara, M. y Torres Herrera, M. (Coords.). (2010). La escuela como organización de conocimiento. México, D. F.: Trillas.

Gore, E. (2003). Conocimiento colectivo. La formación en el trabajo y la generación de capacidades colectivas. Buenos Aires: Granica.

Guns, B. (1996). Aprendizaje organizacional. Naucalpan de Juárez, México: Prentice-Hall Hispanoamericana.

Autor. (2010).

Harvard Business Review. (2000). Gestión del Conocimiento. Bilbao: Deusto.

Koulopoulos, T. y Frappaolo, C. (2000). SMART. Lo fundamental y lo más efectivo acerca de la gerencia del conocimiento. Bogotá: McGRAW-HILL Interamericana.

Martín Fernández, E. (2001). Gestión de instituciones educativas inteligentes. Madrid: McGRAW-HILL Interamericana de España.

Méndez, R. (2000). La Escuela Básica Rural Venezolana ante los Requerimientos de un Entorno Cambiante. Tesis de doctorado no publicada, Universidad Pedagógica Experimental Libertador, Instituto Pedagógico Luis Beltrán Prieto Figueroa, Barquisimeto.

Nonaka, I. y Takeuchi, H. (1999). La organización creadora de conocimiento. México, D. F.: oxford.

Autor. (2001).

Picón Medina, G. (1994). El proceso de convertirse en universidad. Aprendizaje organizacional en la universidad venezolana. Caracas: Fedupel.

Rojas Quiñones, J. M. (2006). Gestión educativa en la sociedad del conocimiento. Bogotá: Cooperativa Editorial Magisterio.

Romero, C. (2004). La escuela media en la sociedad del conocimiento. Buenos Aires: Noveduc.

Senge, P. (1992). La quinta disciplina. Barcelona, España: Granica.

Senge, P., Roberts, C., Ross, R., Smith, B. y Kleiner, A. (2004). La quinta disciplina en la práctica. Buenos Aires: Granica.

Swieringa, J. y Wierdsma, A. (1995). La organización que aprende. Buenos Aires: Addison-Weslwy Iberoamericana.

Wei Choo, C. (1999). La organización inteligente. México, D. F.: Oxford. 